\title{
Comment on: Prevalence, Risk Factors and Assessment of Depressive Symptoms in Patients With Systemic Sclerosis
}

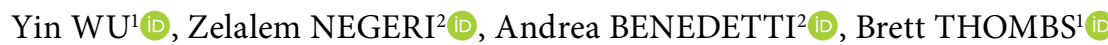 \\ ${ }^{1}$ Department of Psychiatry, Lady Davis Institute for Medical Research, Jewish General Hospital, Montreal, Canada \\ ${ }^{2}$ Department of Biostatistics, and Occupational Health, McGill University, Epidemiology, Montreal, Canada
}

Dr. March et al. ${ }^{1}$ administered the Major Depression Inventory (MDI) to 94 systemic sclerosis (SSc) patients and reported that "the prevalence of depressive symptoms" based on MDI scores of $\geq 20$ was $22.3 \%$, which they described as "high prevalence". Self-report symptom questionnaires like the MDI, however, are not designed to ascertain case status or estimate prevalence and should not be used for this purpose.

Members of our team published studies in 2007-2008 that used questionnaires for this purpose. ${ }^{2,3}$ However, since then we have demonstrated that depression symptom questionnaires tend to overestimate prevalence, sometimes substantially. ${ }^{4,5}$ This is because cutoffs on depression screening questionnaires are typically set to cast a wide net and identify a pool of people who may have depression - but not to ascertain case status. The degree to which estimates of prevalence generated from questionnaires may overestimate depression depends on the questionnaire and cutoff used. Nonetheless, as an example, for the commonly used nine-item Patient Health Questionnaire (PHQ-9) and a standard cutoff of $\geq 10$, sensitivity and specificity are $88 \%$ and $85 \%$, respectively. ${ }^{6}$ Thus, a "prevalence" of $15 \%$ would be generated even if there are no participants with depression. Illustrating this problem further in SSc, Jewett et al. ${ }^{7}$ reported that the 30-day prevalence of major depressive disorder among 345 SSc patients based on a validated diagnostic interview was $3.8 \%$. However, based on the PHQ-9, which was administered simultaneously, and a cutoff of $\geq 10$, the rate was $27 \%,{ }^{6}$ more than seven times the actual prevalence.

The MDI has been used mostly among patients with depressive disorders or those suspected of having depression, and no large primary studies or systematic reviews have established its accuracy for screening or identifying case status among non-psychiatric populations, as used in the study by Dr. March et al. ${ }^{1}$ Thus, it is not known how the percentage of participants with scores of 20 or greater would relate to the percentage who might have a depressive disorder.

Labeling the percentage of patients who score above a threshold on a self-report questionnaire as "prevalence of depressive symptoms" rather

Received: November 14, 2019 Accepted: January 04, 2020 Published online: July 24, 2020

Correspondence: Brett Thombs, MD. Department of Psychiatry, Lady Davis Institute for Medical Research, Jewish General Hospital, H3T 1E4 Montreal, Quebec, Canada. Tel: 5143408222-25112 e-mail: brett.thombs@mcgill.ca Arch Rheumatol 2020;35(3):458-459. 
than depression does not solve the problem. Labeling this as prevalence still clearly indicates that there is some entity that exists and begins at that threshold. However, there is no evidence showing that any cutoff on the MDI separates people into those with significant impairment and those without, which is the purpose of diagnosis. Second, if the objective is simply to identify a threshold where symptoms are present and greater than those below the threshold, any cutoff could be used, rendering any given threshold meaningless in terms of "prevalence".

It is likely the case that people with SSc are more likely to have depression than people without the disease. The percentage reported in the study by Dr. March et al., ${ }^{1}$ however, does not allow us to draw conclusions about the degree that this may be the case.

\section{Declaration of conflicting interests}

The authors declared no conflicts of interest with respect to the authorship and/or publication of this article.

\section{Funding}

Dr. Wu was supported by a Fonds de recherche du Québec - Santé (FRQS) Postdoctoral Training Fellowship. Drs. Benedetti and Thombs were supported by FRQS researcher salary awards.

\section{REFERENCES}

1. March C, Huscher D, Preis E, Makowka A, Hoeppner $\mathrm{J}$, Buttgereit F, et al. Prevalence, Risk Factors and Assessment of Depressive Symptoms in Patients With Systemic Sclerosis. Arch Rheumatol 2019;34:253-61.

2. Thombs BD, Taillefer SS, Hudson M, Baron M. Depression in patients with systemic sclerosis: a systematic review of the evidence. Arthritis Rheum 2007;57:1089-97.

3. Thombs BD, Hudson M, Taillefer SS, Baron M; Canadian Scleroderma Research Group. Prevalence and clinical correlates of symptoms of depression in patients with systemic sclerosis. Arthritis Rheum 2008;59:504-9.

4. Thombs BD, Kwakkenbos L, Levis AW, Benedetti A. Addressing overestimation of the prevalence of depression based on self-report screening questionnaires. CMAJ 2018;190:E44-E49.

5. Levis B, Yan XW, He C, Sun Y, Benedetti A, Thombs BD. Comparison of depression prevalence estimates in meta-analyses based on screening tools and rating scales versus diagnostic interviews: a meta-research review. BMC Med 2019;17:65.

6. Levis B, Benedetti A, Thombs BD; DEPRESsion Screening Data (DEPRESSD) Collaboration. Accuracy of Patient Health Questionnaire-9 (PHQ-9) for screening to detect major depression: individual participant data meta-analysis. BMJ 2019;365:11476.

7. Jewett LR, Razykov I, Hudson M, Baron M, Thombs BD; Canadian Scleroderma Research Group. Prevalence of current, 12-month and lifetime major depressive disorder among patients with systemic sclerosis. Rheumatology 2013;52:669-75. 